\title{
Effect of Polyhydroxybutyrate (PHB) storage on L-arginine production in recombinant Corynebacterium crenatum using coenzyme regulation
}

Meijuan Xu' ${ }^{1}$, Jingru Qin ${ }^{1}$, Zhiming Rao ${ }^{1 *}$, Hengyi You ${ }^{1}$, Xian Zhang ${ }^{1}$, Taowei Yang ${ }^{1}$, Xiaoyuan Wang ${ }^{1}$ and Zhenghong $\mathrm{Xu}^{2^{*}}$

\begin{abstract}
Background: Corynebacterium crenatum SYPA 5 is the industrial strain for L-arginine production. Poly- $\beta$ hydroxybutyrate (PHB) is a kind of biopolymer stored as bacterial reserve materials for carbon and energy. The introduction of the PHB synthesis pathway into several strains can regulate the global metabolic pathway. In addition, both the pathways of PHB and L-arginine biosynthesis in the cells are NADPH-dependent. NAD kinase could upregulate the NADPH concentration in the bacteria. Thus, it is interesting to investigate how both PHB and NAD kinase affect the L-arginine biosynthesis in C. crenatum SYPA 5.

Results: C. crenatum P1 containing PHB synthesis pathway was constructed and cultivated in batch fermentation for $96 \mathrm{~h}$. The enzyme activities of the key enzymes were enhanced comparing to the control strain C. crenatum SYPA 5. More PHB was found in C. crenatum P1, up to $12.7 \%$ of the dry cell weight. Higher growth level and enhanced glucose consumptions were also observed in C. crenatum P1. With respect to the yield of L-arginine, it was $38.54 \pm 0.81 \mathrm{~g} / \mathrm{L}$, increasing by $20.6 \%$, comparing to the control under the influence of PHB accumulation. For more NADPH supply, C. crenatum P2 was constructed with overexpression of NAD kinase based on C. crenatum P1. The NADPH concentration was increased in C. crenatum P2 comparing to the control. PHB content reached $15.7 \%$ and $41.11 \pm 1.21 \mathrm{~g} / \mathrm{L}$ L-arginine was obtained in C. crenatum P2, increased by $28.6 \%$. The transcription levels of key L-arginine synthesis genes, argB, $\arg C, \arg \mathrm{D}$ and $\arg \mathrm{J}$ in recombinant C. crenatum increased 1.9-3.0 times compared with the parent strain.
\end{abstract}

Conclusions: Accumulation of PHB by introducing PHB synthesis pathway, together with up-regulation of coenzyme level by overexpressing NAD kinase, enables the recombinant C. crenatum to serve as high-efficiency cell factories in the long-time L-arginine fermentation. Furthermore, batch cultivation of the engineered $C$. crenatum revealed that it could accumulate both extracellular L-arginine and intracellular PHB simultaneously. All of these have a potential biotechnological application as a strategy for high-yield L-arginine.

Keywords: L-Arginine, Poly-ß-hydroxybutyrate (PHB), NAD kinase, Corynebacterium crenatum SYPA 5

\footnotetext{
*Correspondence: raozhm@jiangnan.edu.cn; zhenghxu@163.com

${ }^{1}$ The Key Laboratory of Industrial Biotechnology of Ministry of Education,

School of Biotechnology, Jiangnan University, Wuxi 214122, Jiangsu,

People's Republic of China

${ }^{2}$ Laboratory of Pharmaceutical Engineering, School of Medicine

and Pharmaceutics, Jiangnan University, Wuxi 214122, Jiangsu, People's

Republic of China
} 


\section{Background}

L-Arginine is a kind of semi-essential amino acid and plays a significant role in nitrogen metabolism and ammonia detoxification as an intermediate in the urea cycle in humans [1]. It is involved in numerous areas of application, such as food flavor, pharmacology and physiology [2, 3]. The biosynthesis of L-arginine in bacteria has become a focus of research interest for the past decades on metabolic regulation. Studies on the L-arginine production have been conducted using the mutants of Corynebacterium, Bacillus and Serratia since the 1960s [4-6]. Several elaborate strategies were designed for efficient production of L-arginine based on the pathways, regulation, and metabolic reaction of amino acids [7, 8]. Corynebacterium crenatum was successfully isolated from soil, and its mutated strain, C. crenatum SYPA 5, is an aerobic, gram-positive, non-sporulating and L-histidine auxotroph industrial bacterium $[9,10]$. In our previous work, much work has been done to increase the production of $\mathrm{L}$-arginine using C. crenatum SYPA 5 as the start strain by adjusting the transfer efficiency for $\mathrm{L}$-arginine, increasing the dissolved oxygen in bacterial, modifying the key enzymes involved in the $\mathrm{L}$-arginine synthesis pathway $[5,10-12]$.

Poly- $\beta$-hydroxybutyrate (PHB), stored as bacterial reserve materials for carbon and energy, is the most popular type of polyhydroxyalkanoate (PHA) that has been well studied in recent years [13]. It is an environmentally friendly biopolymer material due to its prominent properties, such as biodegradability and biocompatibility $[14,15]$. PHB is intracellular while $\mathrm{L}$-arginine is extracellular. It is possible to produce L-arginine and PHB simultaneously, which improves the resource utilization rate. In addition, PHB can provide the cells with carbon source, energy and reducing power, which influence intracellular metabolic flow, oxidation/reduction state and enhance stress resistance of the cells [16]. As reported, the production of several amino acids was enhanced resulting from the extra introduction of the PHB synthesis pathway. In some way, the accumulation of PHB in the cells could be seen as a strategy for amino acids and important metabolic compounds production [17-19]. More the effect of PHB accumulation in the bacterial on L-arginine yield has not been reported yet. During the PHB biosynthesis process, three key enzymes exist including PHB synthase (PhbC), $\beta$-ketothiolase (PhbA) and $\mathrm{NADPH}$-dependent acetoacetyl-CoA reductase (PhbB). Among them, $\mathrm{PhbB}$ is quite special and plays a significant role in PHB synthesis due to its dependence upon NADPH [20]. A high level of NADPH and/or NADPH/NADP ${ }^{+}$ratio has a critical effect on PHB synthesis [21].

During the L-arginine biosynthesis process, the cofactor, like the NADPH concentration, is known to have an important influence on the production by microorganisms because those key enzymes involved in L-arginine biosynthesis require NADPH, such as the NADPH-dependent glutamate dehydrogenase (GdhA) and $\operatorname{ArgC}$ [22]. However, high-yielded L-arginine by enhancing the coenzyme level in the cells has not drawn much attention.

The cofactor pairs NADPH/NADP ${ }^{+}$is essential for all living organisms and plays its important role, mainly in its use as donor and/or acceptor of reducing equivalents in oxidation-reduction reactions in living cells [23]. Many industrially valuable compounds require NADPH for their synthesis and there have been a variety of methods designed to (re) generate this cofactor, like chemical, electrochemical, photochemical, or enzymatic reactions [24, 25]. NADPH can be generated by phosphorylating NAD through NAD kinase. NAD kinase catalyses NAD phosphorylation using ATP and/or inorganic polyphosphate [poly(P)] as phosphoryl donors in the presence of $\mathrm{Mg}^{2+}$ [26]. NAD kinase is ubiquitously distributed from bacteria to human cells and the gene encoding NAD kinase in Escherichia coli, Saccharomyces cerevisiae, and humans have been identified and well-studied [27-30]. Changing the cofactor level by overexpressing the NAD kinase has a positive effect on many industrially valuable compounds, like L-isoleucine in C. glutamicum, isobutanol and thymidine in E. coli [31-33]. As reported, ppnK was the only NAD kinase gene in C. glutamicum and PpnK is essential in the bacteria [34].

Both synthesis pathways of extracellular L-arginine and intracellular PHB are NADPH-dependent. They compete for NADPH, to some degree. Therefore, measures must be taken to improve the cofactor level. It is interesting to see the effect of NAD kinase overexpression on the PHB and $\mathrm{L}$-arginine yield in C. crenatum, together with the effect of PHB on L-arginine yield. In this study, we were intended to construct a high-yield $\mathrm{L}$-arginine strain by introducing the PHB synthesis pathway using C. crenatum SYPA 5 as the start strain. Meanwhile, the NAD kinase, PpnK, was overexpressed to balance the cofactor level. Furthermore, batch cultivation of the engineered C. crenatum revealed that it was able to accumulate both extracellular L-arginine and intracellular PHB simultaneously.

\section{Results and discussion}

\section{Construction of C. crenatum P1 and C. crenatum P2}

$\mathrm{PHB}$, the best-known polyhydroxyalkanoates (PHA), has been reported to influence intracellular metabolic flow, oxidation/reduction state [18] and enhance stress resistance of the host [16], as well. In this study, pDP10, containing the PHB synthesis genes, phbCAB, from Ralstonia eutropha, was introduced into C. crenatum SYPA 5 to generate C. crenatum P1 (Fig. 1). As depicted in Fig. 2, L-arginine metabolic pathway started from acetylCoA to $\alpha$-ketoglutarate through TCA cycle, and then L-glutamate was formed with GdhA. Finally, L-arginine was produced by the catalysis of a series of enzymes encoded by the $\operatorname{argCJBDFRJH}(\operatorname{argC} \sim \mathrm{H})$ cluster involved 

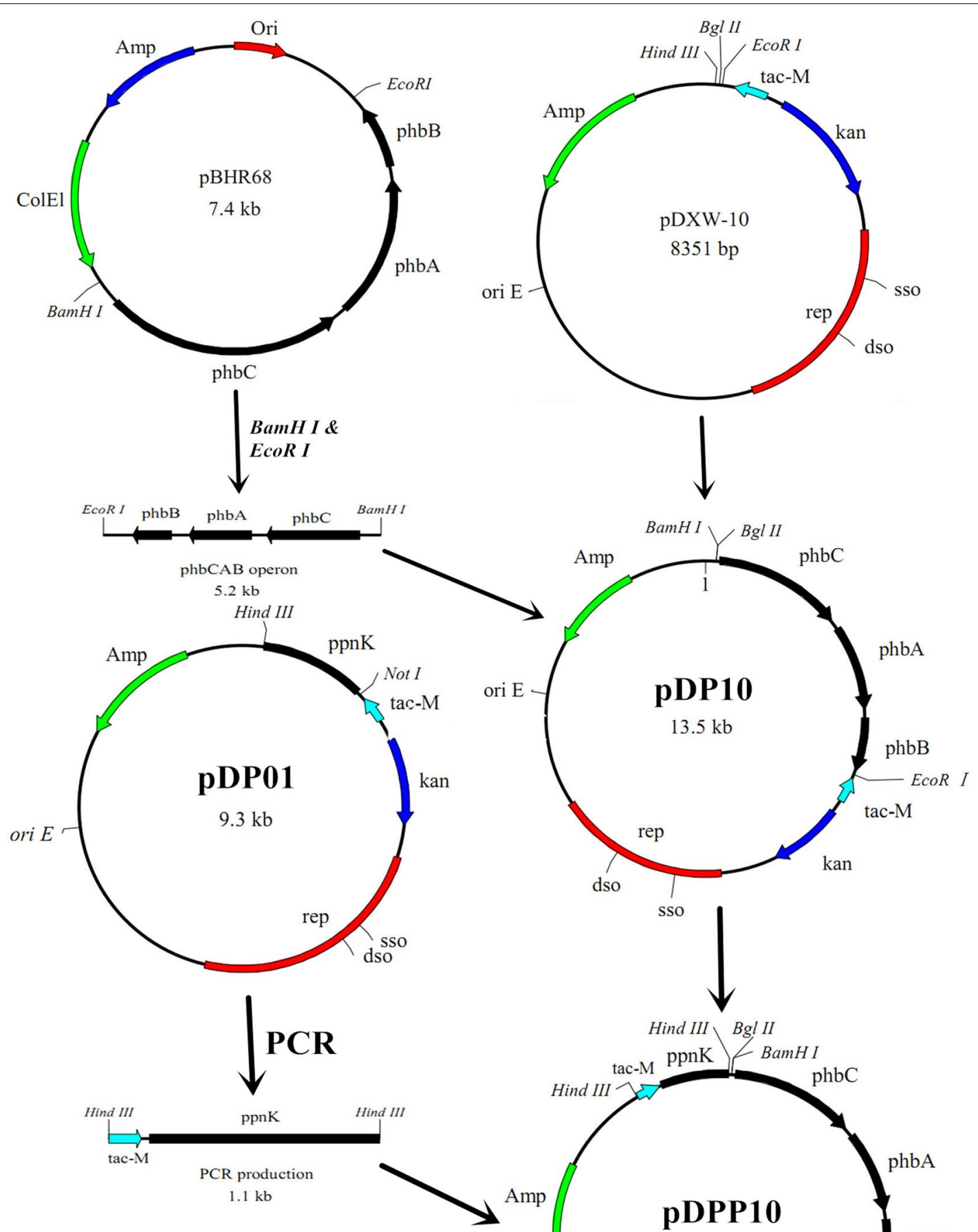

Hind III Bgl II

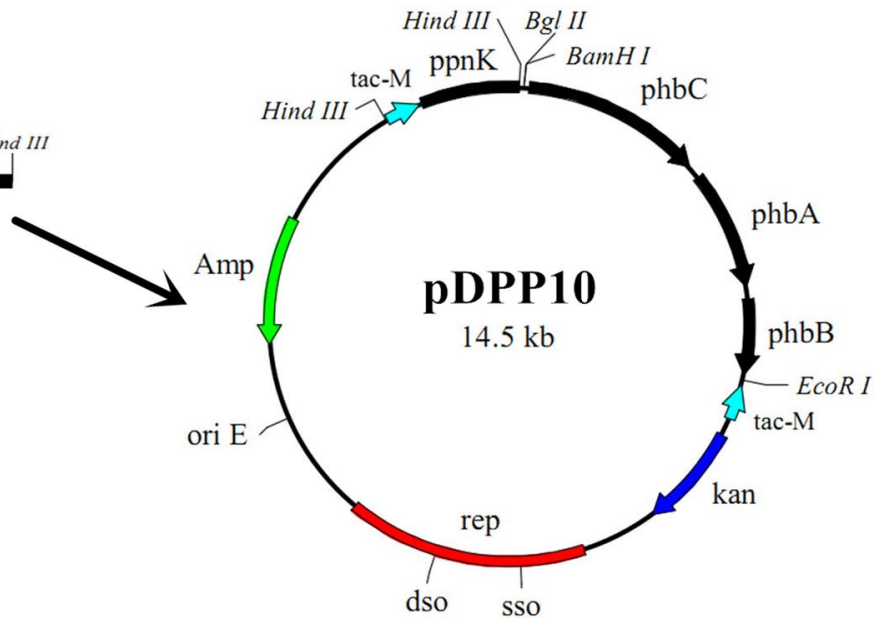

Fig. 1 Construction of the plasmids in this study 


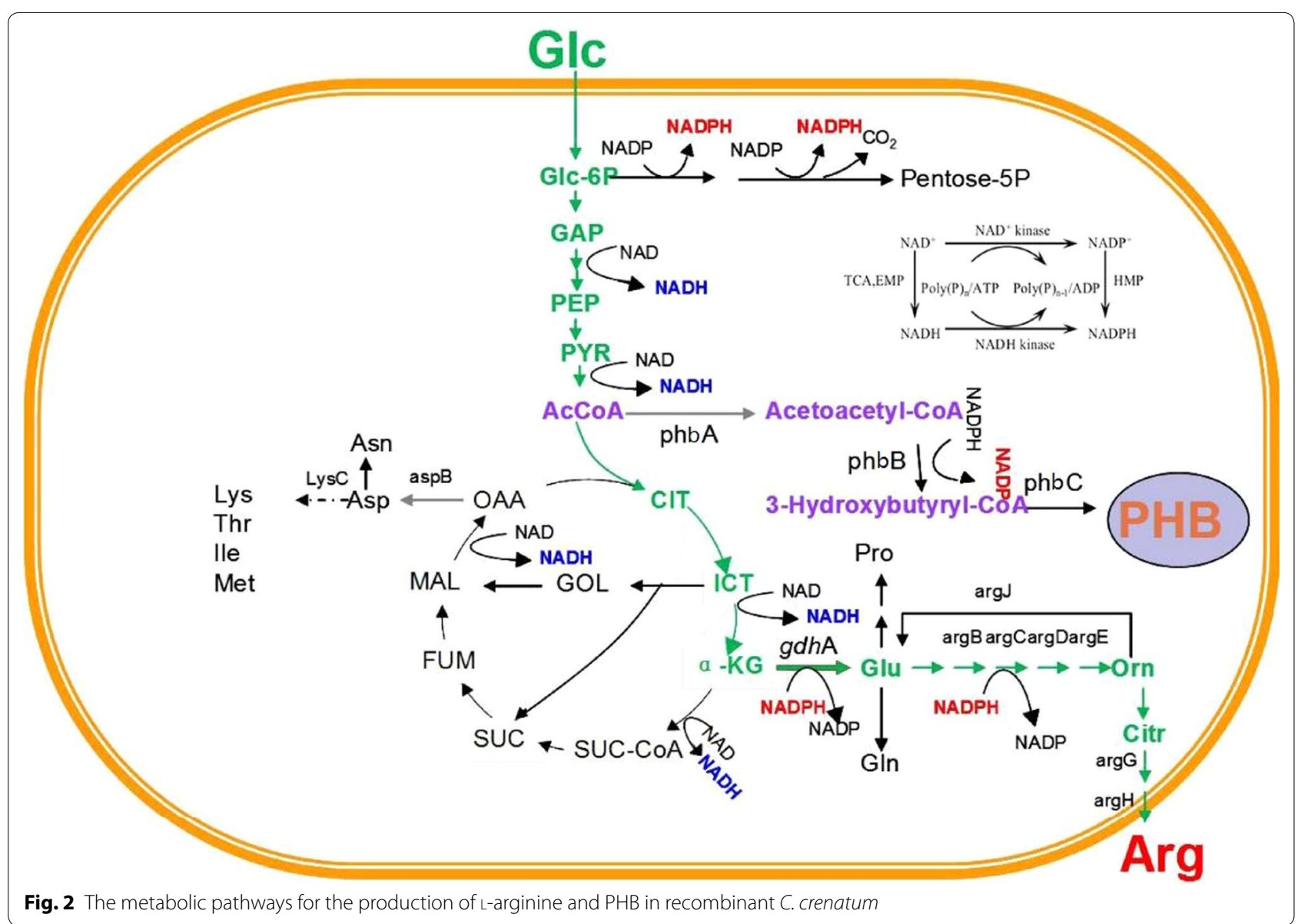

in L-arginine biosynthesis. On the other hand, PHB was formed from acetyl-CoA with the three key enzymes, PHB synthase (PhbC), $\beta$-ketothiolase (PhbA) and $\mathrm{NADPH}$-dependent acetoacetyl-CoA reductase (PhbB).

Sources of NADPH in bacteria mainly contain the pentose phosphate pathway (PPP), isocitrate dehydrogenase in the tricarboxylic acid (TCA) cycle and the transhydrogenase system [23]. As illustrated in Fig. 2, NADPH was necessary in both the L-arginine and PHB metabolic pathways. PHB synthesis was a NADPH-dependent process for $\mathrm{PhbB}$ worked under the existence of $\mathrm{NADPH}$ and during the L-arginine metabolic pathways, GdhA and ArgC also required NADPH. In addition, in previous studies with arginine fermentation in C. crenatum SYPA 5, too much NADH can reduce the metabolic flow of the glycolytic pathway and increase the by-products (lactate and acetate) concentration [9]. As reported, in C. glutamicum, the NAD kinase (PpnK) regulated the cofactor level and could enhance the NADPH concentration [34]. Differential expression of $p p n K$ has not been reported to date and $p p n K$ might be essential and the only NAD kinase gene in Corynebacterium sp. [35]. Therefore, overexpressing homologous NAD kinase by cloning and amplifying the homologous $p p n K$ gene in C. crenatum P1 deserved an attempt. We developed an approach to increase the NADPH availability in vivo through introducing NAD kinase, the key NADPH producing enzyme in C. crenatum, and were intended to see whether it had any effect on the L-arginine and PHB production or not. C. crenatum P2, containing the pDPP10, was created by introducing PpnK on the basis of C. crenatum P1.

\section{Enzyme activities assay of PhbC, PhbA, PhbB and PpnK}

In this study, the $p h b C A B$ cluster of Ralstonia eutropha was introduced into C. crenatum SYPA 5. In order to verify the activity of the over-expressed PhbC, PhbA and PhbB from the $C$. crenatum $\mathrm{PHB}$ production strains, $C$. crenatum SYPA 5, C. crenatum P1 and C. crenatum P2 were cultivated in LBG medium (LB with $0.5 \%$ glucose) for $24 \mathrm{~h}$ and then the crude enzyme activities of PhbC, PhbA and PhbB were detected (Table 1). The synthesis of bacterial PHB was dependent on the expression and activity of a key enzyme, PHB synthase (PhbC). Therefore, enhancing the activity of PhbC was a good way to increase PHB content [36]. To investigate the activity of $\mathrm{PhbC}, 3 \mathrm{HB}-\mathrm{CoA}$ was used as the 
Table 1 Assay of enzyme activities of crude PhbC, PhbA, PhbB and PpnK in C. crenatum SYPA 5, P1 (SYPA 5/phbCAB) and P2 (SYPA 5/phbCAB-ppnK)

\begin{tabular}{llllr}
\hline Strains & \multicolumn{2}{l}{ Specific enzyme activities } & & \\
\cline { 2 - 5 } & PhbC (U/mg) & PhbA (U/mg) & PhbB (U/mg) & ATP-NAD kinase (U/g) \\
\hline C. crenatum SYPA 5 & $0.02 \pm 0.00$ & $0.02 \pm 0.00$ & $0.06 \pm 0.01$ & $0.63 \pm 0.03$ \\
C. crenatum P1 & $0.27 \pm 0.01$ & $0.58 \pm 0.02$ & $0.64 \pm 0.02$ & $0.67 \pm 0.04$ \\
C. crenatum P2 & $0.29 \pm 0.01$ & $0.61 \pm 0.03$ & $0.83 \pm 0.03$ & $84.35 \pm 0.41$ \\
\hline
\end{tabular}

Samples were taken at $24 \mathrm{~h}$ of the shake flask using LBG culture. ATP-NAD kinase contained ATP-NAD ${ }^{+}$kinase and ATP-NADH kinase while PolyP-NAD kinase contained PolyP-NAD ${ }^{+}$kinase and PolyP-NADH kinase. Each data represented the average value of three independent measurements

substrate, and the release of CoA during polymerization was measured to determine the total enzyme activity [37]. The total activity of PhbC was measured using the soluble fraction of the crude extract. The total synthase activity of cell extracts containing $\mathrm{PhbC}$ in recombinant $C$. crenatum was about $0.28 \mathrm{U} / \mathrm{mg}$ while it was quite low, $0.02 \mathrm{U} / \mathrm{mg}$, in C. crenatum SYPA 5. As the first enzyme in the PHB synthesis pathway, the regulatory role of $\beta$-ketothiolase (PhbA) has been extensively discussed. PhbA from different strains differed with respect to the effect of concentrations of acetoacetyl-CoA or NADH and NADPH on the thiolysis reaction [38]. The enzyme activities of PhbA in C. crenatum P1 and C. crenatum P2 showed a significant increase, 30-fold, compared to the C. crenatum SYPA 5. The effect of about 11-fold enhancement in recombinant C. crenatum was found in the activities of NADPH-dependent acetoacetyl-CoA reductase (PhbB). PhbB was special among the three key PHB synthesis enzymes due to its coenzyme dependency upon NADPH. Therefore, the concentration of NADPH has quiet influence on the $p h b B$ expression. The high activity of PHB synthesis related enzymes in recombinant strains could be associated with their elevated level of genes expression through introducing the exogenous plasmid pDP10 or pDPP10.

NAD kinase, PpnK, was the key enzyme for the biosynthesis of $\mathrm{NADP}^{+}$and NADPH in C. crenatum and it was critical for the generation of NADPH [39]. It could be divided into two kinds according to the phosphoryl acceptor. The enzyme that phosphorylates only $\mathrm{NAD}^{+}$to form $\mathrm{NADP}^{+}$was termed NAD ${ }^{+}$kinase (EC 2.7.1.23), and the enzyme that phosphorylates both $\mathrm{NAD}^{+}$and $\mathrm{NADH}$ to form $\mathrm{NADP}^{+}$and $\mathrm{NADPH}$ is $\mathrm{NADH}$ kinase (EC 2.7.1.86) [40, 41]. So far, the NAD kinases characterized either use ATP and PolyP as phosphoryl donors or were solely active with ATP [34] and PpnK here was the former kind. In order to investigate whether the $p p n K$ gene expressed well in $C$. crenatum P2, the NAD kinase activity was determined and compared to the control C. crenatum SYPA 5. In the crude cell extract from the $p p n K$-expressing strain, the ATP-dependent and PolyP-dependent NAD kinase activity increased approximately 130 -fold $(84.35 \pm 0.41 \mathrm{U} / \mathrm{g})$ and 16-fold $(3.12 \pm 0.02 \mathrm{U} / \mathrm{g})$, respectively, compared to the control C. crenatum SYPA $5(0.63 \pm 0.03 \mathrm{U} / \mathrm{g})$ and $(0.18 \pm 0.01 \mathrm{U} / \mathrm{g})$ (Data showed in Additional file 1). From the above observations, these differences in enzyme activities reflected the different expression levels of the PpnK proteins in these microorganisms, which might be due to the existence of the strong tac-M promoter [42].

\section{The effect of PHB accumulation on L-arginine operon transcription}

In order to investigate the effect of PHB accumulation on gene transcription, RT-PCR was performed. In this experiment, we selected $\arg B, \arg C, \arg D, \operatorname{argF}, \arg G, \operatorname{argH}$ and argJ gene as the experimental target since they directly involved in the L-arginine production. We found that the transcription levels of four L-arginine operon genes, $\operatorname{argB}$, $\arg C$, $\arg D$ and $\arg J$ in $C$. crenatum P1 increased 1.9-3.0 times compared with the parent strain (Fig. 3). Although the transcription of other genes in C. crenatum P1 decreased slightly, this result at least proved that the intracellular PHB accumulation enhanced the transcription levels of several L-arginine key synthesis genes. At the same time, the phb$\mathrm{CAB}$ overexpression in C. crenatum $\mathrm{P} 1$ caused a dramatic

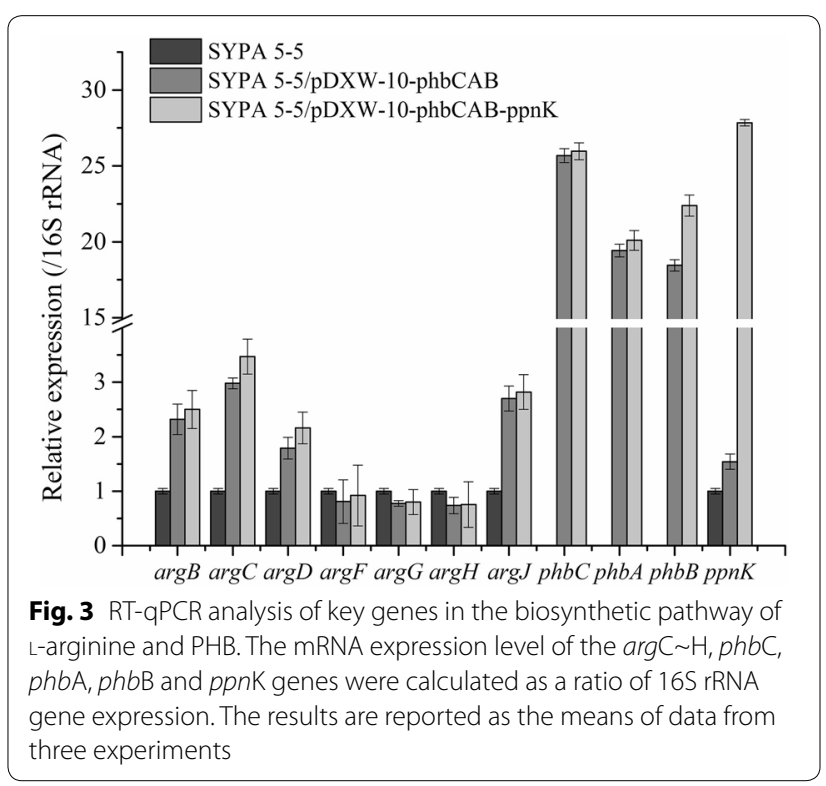


up-regulation of $p p n \mathrm{~K}$ transcription. In C. crenatum $\mathrm{P} 2$, a similar data were also obtained for the $p h b \mathrm{CAB}$ and $p p n \mathrm{~K}$ gene which an increase in gene expression corresponded to an increase in enzyme activities. Thus, the introduction of the PHB synthesis pathway and NAD kinase overexpression affected the transcription of key genes of the L-arginine biosynthesis pathway. Obviously, the $p h b \mathrm{C}$ and $p p n \mathrm{~K}$ gene under the control of the promoters $\mathrm{P} p h b C$ and tacM caused the increased transcription of the two genes. The two genes $p h b \mathrm{~B}$ and phbA followed the same trend, but it was much less pronounced. Consequensely, L-arginine yield increasing effect in recombinant $C$. crenatum was explained at the transcriptional level.

\section{TEM imaging}

In order to verify the expression effect of recombinant plasmid pDXW-10-phbCAB more directly, C. crenatum SYPA 5 and its recombinants were prepared for TEM analysis. The results were exhibited in Fig. 4. From Fig. 4, (a) showed C. crenatum SYPA 5 with an extremely small amount of PHB granules; (b) displayed the PHB granules in the recombinant SYPA 5/pDXW-10-phbCAB. Due to the $\mathrm{PHB}$ gene cluster expressing well in the strain, $\mathrm{PHB}$ granules could be seen obviously; thanks to NAD kinase expression in C. crenatum, the competition environment of the NADPH was eased in the process of L-arginine and PHB biosynthesis. As it is shown in (c), the PHB existed evidently in the cell, more than that of the strain with $\mathrm{PHB}$ synthesis operon only. Thus, we conclude that, the NAD kinase in the recombinant $C$. crenatum was overexpressed.

\section{Effect of PHB accumulation on L-arginine production by $C$. crenatum $\mathrm{P} 1$ in 5 -I fermentor}

To find the effect of PHB accumulation on L-arginine production, the growth of the strains C. crenatum SYPA 5 and $C$. crenatum P1 were compared under batch cultivation condition. From Fig. 5, it was easy to find that the PHB content in C. crenatum SYPA 5 was low, varied below $3.0 \%$, while in C. crenatum P1, the PHB content increased to $12.7 \%$, about fourfold, at maximum and more $\mathrm{PHB}$ accumulated in the $C$. crenatum $\mathrm{P} 1$. The recombinant $C$. crenatum $\mathrm{P} 1$ demonstrated a superior ability in growth compared to that of the control C. crenatum SYPA 5 with the final $\mathrm{OD}_{562}$ reaching 81 at $96 \mathrm{~h}$. However, the glucose consumption of the recombinant strain was more than that of the control strain. Besides these differences, the recombinant strain exhibited similar L-arginine production with the control strain in the early stage of the culture. Notably, the large gap in $\mathrm{L}$-arginine production appeared after $32 \mathrm{~h}$ comparing to the control. The final concentration of $\mathrm{L}$-arginine of $C$. crenatum P1 was $38.54 \pm 0.81 \mathrm{~g} / \mathrm{L}$, increasing by $20.6 \%$ comparing to C. crenatum SYPA $5(31.95 \pm 0.68 \mathrm{~g} / \mathrm{L})$. Meanwhile, the metabolic intermediate and by-product, $\alpha$-ketoglutarate, acetate and lactic acid (Table 2) and some other amino acids (Table 3) in batch cultivation were also analyzed to investigate the effect of PHB accumulation on C. crenatum metabolism. These data show that, during the fermentation, the formation of some other amino acids except for L-arginine and L-glycine in C. crenatum P1 were apparently less than that in the $C$. crenatum SYPA 5 . However, the concentration of acetate and lactic acid increased.

As reported, the expression of PHB synthesis genes, which increased L-glutamate production with 39-68\% in shake flask and $23 \%$ in fermentor, had a positive effect on glutamate production in C. glutamicum [18]. PHB also had a global effect on the host in L-tryptophan producing strain and upregulated the transcription of a tryptophan operon, leading to improvement of the L-tryptophan production [17]. Given these results, it was assumed that the introduction of the PHB synthesis pathway into the cells would affect microbial global metabolism, leading to a difference
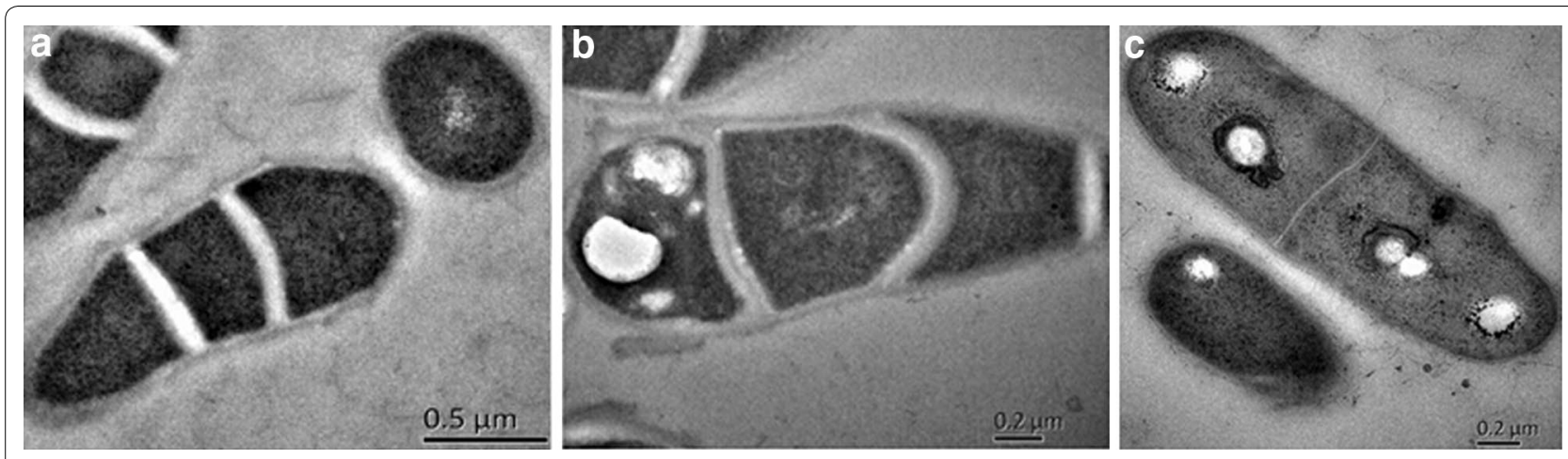

Fig. 4 TEM images of $C$. crenatum SYPA 5 and the recombinant C. crenatum P1(SYPA 5/phbCAB) and P2 (SYPA 5/phbCAB-ppnK) biomasses. Bacteria appear in gray and PHB vesicles in white a C. crenatum SYPA 5; b C. crenatum P1; c C. crenatum P2 

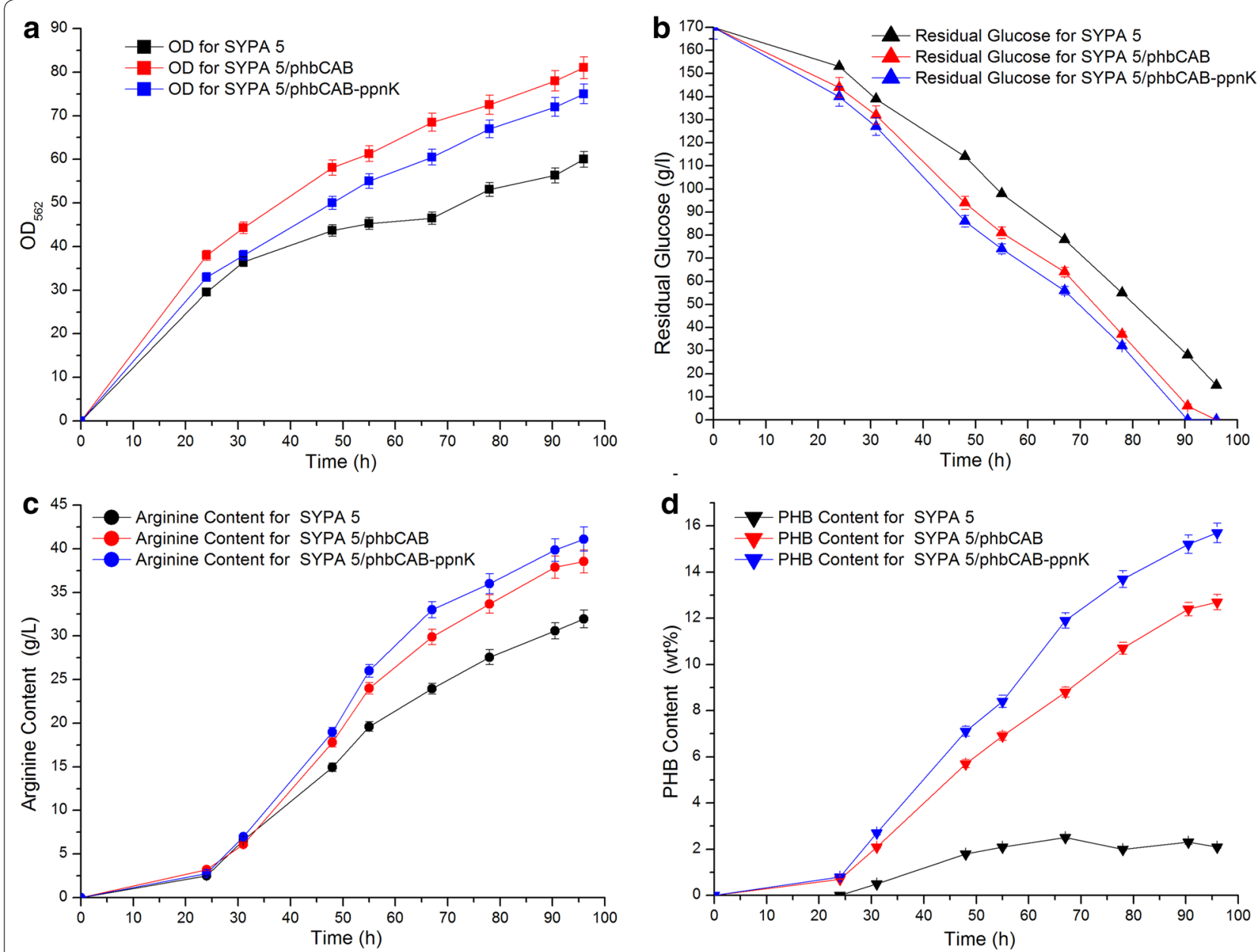

Fig. 5 Batch fermentation profile. Comparisons of arginine production between the wild-type C. crenatum SYPA 5 and the recombinant C. crenatum P1 (SYPA 5/phbCAB) and P2 (SYPA 5/phbCAB-ppnK) in fermentation media. a OD 562 . (Black filled square) C. crenatum SYPA 5; (Red filled square) C. crenatum P1; (Blue filled square) C. crenatum P2. b Residual Glucose. (Black filled triangle) C. crenatum SYPA 5; (Red filled triangle) C. crenatum P1; (Blue filled triangle) C. crenatum P2. c L-arginine. (Black filled circle) C. crenatum SYPA 5; (Red filled circle) C. crenatum P1; (Blue filled circle) C. crenatum P2. d PHB content. (Black filled inverted triangle) C. crenatum SYPA 5; (Red filled inverted triangle) C. crenatum P1; (Blue filled inverted triangle) C. crenatum P2

in the formation of some products, such as some kind of amino acids. In this study, due to the exogenous plasmid pDP10 harboring the PHB synthesis genes, phbCAB, in $C$. crenatum $\mathrm{P} 1$, the recombinant strain grew faster even though the glucose metabolism was enhanced. At the same time, the significant metabolite intermediate for $\mathrm{L}$-arginine production, $\alpha$-ketoglutarate (from $0.64 \pm 0.03 \mathrm{~g} / \mathrm{L}$ to $0.96 \pm 0.02 \mathrm{~g} / \mathrm{L}$ ), had a slight increase flux in the recombinant strain. More bacterial and more metabolic precursor might result in more $\mathrm{L}$-arginine production than that in the parent strain. Moreover, the existence of PHB in the cells might enhance stress resistance of the host and protect the cells, to some degree. Still, more glucose was consumed and conserved in the cell at the cost. It was obvious that the accumulation of PHB affecting the productivity of the long L-arginine fermentation. This might be a major reason why overexpressed $p h b C A B$ could evolve significant L-arginine yield.

PHB accumulation in the bacteria influenced and regulated the global pathways, including the co-factor level. The NADPH pool in the cells had a slight increase, from $35 \pm 2 \mathrm{pmol} / \mathrm{OD}_{562}$ to $39 \pm 2 \mathrm{pmol} /$ $\mathrm{OD}_{562}$, in C. crenatum P1 (Table 4), this was likely why more $\mathrm{L}$-arginine was yielded. However, considering that the competitive relationship between $\mathrm{PHB}$ and $\mathrm{L}$-arginine production on $\mathrm{NADPH}$, it is interesting to take measures to regulate the cofactor level in the cells in order to see whether any further positive effect on the L-arginine yield exists. 
Table 2 Concentrations of acetate and lactic acid in batch cultivation of C. crenatum SYPA 5, P1 (SYPA 5/phbCAB) and P2 (SYPA 5/phbCAB-ppnK)

\begin{tabular}{|c|c|c|c|c|c|}
\hline \multirow[t]{2}{*}{ By-products } & \multicolumn{5}{|c|}{ Concentration (g/L) } \\
\hline & $24 \mathrm{~h}$ & $48 \mathrm{~h}$ & $60 \mathrm{~h}$ & $84 \mathrm{~h}$ & $96 \mathrm{~h}$ \\
\hline \multicolumn{6}{|l|}{ Acetate } \\
\hline C. crenatum SYPA 5 & $2.02 \pm 0.03$ & $1.74 \pm 0.02$ & $1.50 \pm 0.01$ & $0.82 \pm 0.02$ & $0.74 \pm 0.01$ \\
\hline C. crenatum P1 & $2.13 \pm 0.03$ & $1.81 \pm 0.02$ & $1.69 \pm 0.02$ & $1.37 \pm 0.02$ & $1.18 \pm 0.01$ \\
\hline C. crenatum P2 & $2.08 \pm 0.04$ & $1.88 \pm 0.02$ & $1.46 \pm 0.02$ & $1.01 \pm 0.02$ & $0.94 \pm 0.02$ \\
\hline \multicolumn{6}{|l|}{ Lactic acid } \\
\hline C. crenatum SYPA 5 & $2.26 \pm 0.03$ & $1.80 \pm 0.02$ & $1.35 \pm 0.02$ & $1.07 \pm 0.02$ & $0.86 \pm 0.01$ \\
\hline C. crenatum P1 & $2.37 \pm 0.03$ & $1.97 \pm 0.03$ & $1.65 \pm 0.02$ & $1.76 \pm 0.02$ & $1.95 \pm 0.02$ \\
\hline C. crenatum P2 & $2.21 \pm 0.01$ & $1.78 \pm 0.03$ & $1.29 \pm 0.02$ & $1.37 \pm 0.02$ & $1.50 \pm 0.02$ \\
\hline
\end{tabular}

Each data represented the average value of three independent measurements

Table 3 Production of other related amino acids by $C$. crenatum SYPA 5, P1 (SYPA 5/phbCAB) and P2 (SYPA 5/phbCAB-ppnK)

\begin{tabular}{llll}
\hline Amino acids & \multicolumn{3}{l}{ Concentration $\mathbf{( g / L )}$} \\
\cline { 2 - 4 } & C. crenatum SYPA 5 & C. crenatum P1 & C. crenatum P2 \\
\hline Ile & $2.62 \pm 0.00$ & $2.34 \pm 0.00$ & $3.60 \pm 0.01$ \\
Lys & $4.39 \pm 0.01$ & $4.09 \pm 0.01$ & $5.04 \pm 0.02$ \\
Glu & $1.03 \pm 0.02$ & $0.73 \pm 0.00$ & $0.51 \pm 0.00$ \\
Gly & $0.30 \pm 0.00$ & $0.45 \pm 0.00$ & $0.23 \pm 0.00$ \\
Thr & $0.29 \pm 0.00$ & $0.19 \pm 0.00$ & $0.17 \pm 0.00$ \\
Val & $0.56 \pm 0.00$ & $0.42 \pm 0.00$ & $0.38 \pm 0.00$ \\
Orn & $0.52 \pm 0.00$ & $0.26 \pm 0.00$ & $0.26 \pm 0.00$ \\
Ser & $0.21 \pm 0.00$ & $0.10 \pm 0.00$ & $0.10 \pm 0.00$ \\
\hline
\end{tabular}

The samples were taken at $96 \mathrm{~h}$ of the batch fermentation in 5-I fermentor. Each data represented the average value of three independent measurements

\section{Effect of PpnK introduction on L-arginine production}

As reported, the impact of $p p n K$ overexpression on lysine production was positive in C. glutamicum [34], so was isoleucine production in C. glutamicum [39] and thymidine production in E. coli [33]. In this study, we found that PHB accumulated in recombinant C. crenatum P1 and that $\mathrm{L}$-arginine production increased by $20.6 \%$ due to the accumulation of PHB (Fig. 5). Furthermore, NADPH pool played an important role in $\mathrm{PHB}$ and $\mathrm{L}$-arginine production. Therefore, it was interesting to see how the extra introduction of NAD kinase into C. crenatum P1 affected $\mathrm{PHB}$ and L-arginine biosynthesis. In the bacteria, the reducing power $[\mathrm{H}]$ was mainly generated by EMP, HMP and TCA cycle while the formation of PHB and L-arginine consumed $[\mathrm{H}]$, which keep the oxidation/ reduction stateed balanced. The overexpression of PpnK increased the $\left(\mathrm{NADP}^{+}+\mathrm{NADPH}\right) /\left(\mathrm{NAD}^{+}+\mathrm{NADH}\right)$ rate by fourfold in $C$. crenatum $\mathrm{P} 2$ comparing to that in C. crenatum SYPA 5. As consequence, there was more NADPH supply for PHB synthesis, accounting for $15.7 \%$ of increased PHB at max while $12.7 \%$ in C. crenatum P1 (Fig. 5). Excess amount of NAD kinase could be inhibited by the regulation mechanism, like $\mathrm{NADP}^{+}$[43]. In our investigation, two more exogenous genes existed in the cells. Both of these could contribute to the decrease in $\mathrm{OD}_{562}$ in C. crenatum $\mathrm{P} 2$ comparing to that in C. crenatum P1. However, the $\mathrm{OD}_{562}$ in C. crenatum P2 was still above that in $C$. crenatum SYPA 5 and this might be due to the PHB synthesis genes existed (Fig. 5). The concentration of $\alpha$-ketoglutarate exhibited no much difference between C.crenatum SYPA 5 and C. crenatum P2 in the early stage but a slight increase in C. crenatum P2

Table 4 Concentrations of intracellular NAD ${ }^{+}$, NADH, NADP ${ }^{+}$and NADPH in C. crenatum SYPA 5, P1 (SYPA 5/phbCAB) and P2 (SYPA 5/phbCAB-ppnK)

\begin{tabular}{|c|c|c|c|c|c|}
\hline \multirow[t]{2}{*}{ Strains } & \multicolumn{5}{|c|}{ Concentration (pmol/OD ${ }_{562}$ ) } \\
\hline & $\mathrm{NAD}^{+}$ & $\mathrm{NADH}$ & NADP $^{+}$ & NADPH & $\left(\mathrm{NADP}^{+}+\mathrm{NADPH}\right) /\left(\mathrm{NAD}^{+}+\mathrm{NADH}\right)$ \\
\hline C. crenatum SYPA 5 & $571 \pm 42$ & $61 \pm 4$ & $161 \pm 9$ & $35 \pm 2$ & 0.31 \\
\hline C. crenatum P1 & $366 \pm 27$ & $70 \pm 5$ & $127 \pm 8$ & $39 \pm 2$ & 0.38 \\
\hline C. crenatum P2 & $163 \pm 10$ & $65 \pm 4$ & $205 \pm 14$ & $71 \pm 5$ & 1.21 \\
\hline
\end{tabular}

The samples were taken at $96 \mathrm{~h}$ of the batch fermentation in 5-I fermentor. Each data represented the average value of three independent measurements 
after $84 \mathrm{~h}$ (data was not shown). Higher growth level and stronger pathway from $\alpha$-ketoglutarate to glutamate due to more NADPH supply in C. crenatum P2 would be why the L-arginine production was higher than that in C. crenatum P1, reaching $41.11 \pm 2.11 \mathrm{~g} / \mathrm{L}$ (Fig. 5). In addition, less production of some other amino acids in C. crenatum P2 but Ile and Lys, which were quiet NADPH-dependent and increased by 53.8 and $23.2 \%$ comparing to that of $C$. crenatum P1, represently, also contributed to the high production of $\mathrm{L}$-arginine (Table 3 ). With respect to the concentration of by-products, acetate and lactic acid in C. crenatum P2 were lower than those in P1 because of the enhancement of the ratio of $\left(\mathrm{NADP}^{+}+\mathrm{NADPH}\right) /$ $\left(\mathrm{NAD}^{+}+\mathrm{NADH}\right)$ due to the overexpression of ppnK (Table 2). All of these could also explain why more L-arginine was produced. The concentration of NADPH was increased by onefold resulted from PpnK overexpression in C. crenatum P2 comparing to C. crenatum SYPA 5 (Table 4), which enhanced the metabolism, to some degree, especially in those NADPH-dependent pathways. That would be the reason why the glucose consumption rate in C. crenatum P2 was faster than both of that in C.crenatum SYPA 5 and C. crenatum P1 (Fig. 5). Accordingly, increasing the glucose consumption resulted in a higher glucose-to-L-arginine conversion. The C. crenatum P2 showed the slight improvement in the glucoseL-arginine conversion with a $14.2 \%$ increase, compared to the parent strain C. crenatum SYPA 5(24.1 g Arg/100 g Glc vs. $21.1 \mathrm{~g}$ Arg/100 g Glc). Besides improving the conversion of glucose to L-arginine, employing the PHB synthesis pathway led to an increase of glucose to PHB. The intracellular PHB content began to sharply increase to $15.7 \%$ upon the overexpression of phbCAB operon in the culture broth of $C$. crenatum P2. Taken together, the more glucose utilization have been achieved by introducing PHB synthesis pathway, together with up-regulation of coenzyme level by overexpressing NAD kinase in $C$. crenatum SYPA 5.

\section{Conclusions}

In conclusion, in $C$. crenatum recombinants harboring the phbCAB cluster, more glucose was consumed and conserved in the cell at the cost. The existence of PHB in the cells might enhance stress resistance of the host and protect the cells. Obviously, by employing the PHB synthesis pathway, the L-arginine productivity was increased during the late stage of high-yield L-arginine fermentation. Therefore, it was optimistic that the accumulation of PHB affecting the productivity of the longtime L-arginine fermentation. Meanwhile, the high-yield $\mathrm{L}$-arginine recombinant $C$. crenatum $\mathrm{P} 2$ was constructed by overexpressing the NAD kinase encoding gene $p p n \mathrm{~K}$ into C. crenatum P1. Overexpressing the NAD kinase also enhanced these effects. Furthermore, batch cultivation of the engineered C. crenatum revealed that it could accumulate both extracellular L-arginine and intracellular PHB simultaneously. With those strategies, the recombinant $C$. crenatum with PHB accumulation and NAD kinase overexpression could increase the concentration of NADPH in the coenzyme pool of the cell and serve as high-efficiency cell factories for $\mathrm{L}$-arginine production.

\section{Methods}

\section{Bacterial strains and plasmids}

All the bacterial and plasmids used in the study are listed in Table 5.

\section{Construction of plasmids}

The $p h b C A B$ operon was obtained by double-digesting the plasmid pBHR68 with EcoR I and $B a m H$ I. It was then ligated into the $E$. coli-C. crenatum shuttle expression vector, pDXW-10, to generate the recombinant plasmid pDP10. The native promoter of the $p h b C$ gene was deleted and the native ribosome binding site (RBS) was changed with a consensus RBS sequence AAAGGAGGGAAATC of highly expressed gene. To construct pDPP10, two steps were done. Firstly, ppnK gene from C. crenatum SYPA 5 was amplified by primers (5'-ATTTGCGGCCGC AAAGGAGGGAAATC ATGACTGCACCCACGAA-3') and (5'-CCCAAGCTT TTACCCCGCTGACCTGG-3') using C. crenatum SYPA 5 genome as a temple (the two primers above was designed using the $p p n \mathrm{~K}$ gene of C. glutamicum ATCC 13032). Then the PCR product was digested by NotI and HindIII and inserted into Not I-Hind III sites of pDXW10. All of these resulted in the pDP01. Secondly, the pDP01 was used as the temple and the tacM-ppnK site was amplified by primer $5^{\prime}$-CCCAAGCTTTTACCCCG CTGACCTGG-3' $3^{\prime}$ and $5^{\prime}$ - CCCAAGCTTTCGGAAGCT GTGGTATGG- ${ }^{\prime}$. The PCR product was inserted into pDP10 with the Hind III digestion site after dephosphorylation using CIAP. Both of the pDP10 and pDPP10 were transferred into C. crenatum SYPA 5, generating C. crenatum $\mathrm{P} 1$ and C. crenatum $\mathrm{P} 2$.

\section{Cultivation medium and conditions}

LBG medium (LB with $0.5 \%$ glucose) was used for seed culture preparation supplemented with appropriate antibiotic (kanamycin, $25 \mathrm{mg} / \mathrm{L}$ ). The fermentation medium contained the following $(\mathrm{g} / \mathrm{L})$ : glucose $170,\left(\mathrm{NH}_{4}\right)_{2} \mathrm{SO}_{4} 20$, yeast extract $12, \mathrm{MgSO}_{4} \cdot 7 \mathrm{H}_{2} \mathrm{O} 0.5, \mathrm{KCl} 1, \mathrm{KH}_{2} \mathrm{PO}_{4} 1.5$, $\mathrm{FeSO}_{4} \cdot 7 \mathrm{H}_{2} \mathrm{O} 0.02, \mathrm{MnSO}_{4} \cdot \mathrm{H}_{2} \mathrm{O} 0.02(\mathrm{pH}$ 7.0).

The seed cultures were incubated at $30{ }^{\circ} \mathrm{C}$ in LBG for $24 \mathrm{~h}$ at $160 \mathrm{rpm}$ on a rotary shaker and then $125 \mathrm{ml}$ seed culture was incubated into the fermentation medium (inoculated with $5 \% \mathrm{v} / \mathrm{v}$ seed culture)for the 5-1 
Table 5 Strains and plasmids used in this study

\begin{tabular}{|c|c|c|}
\hline Strains and plasmids & Relevant characteristics & References \\
\hline Corynebacterium crenatum SYPA 5 & L-Arginine production bacterium & {$[12]$} \\
\hline C. crenatum P1 & Derived from C. crenatum SYPA 5, Harboring pDP10 & This study \\
\hline C. crenatum P2 & Derived from C. crenatum SYPA 5, harboring pDPP10 & This study \\
\hline pBHR68 & pBluescript SK-derivative, containing the entire phbCAB operon of Ralstonia eutropha $\mathrm{H} 16$ & {$[44]$} \\
\hline pDXW-10 & E. coli-C. crenatum shuttle expression vector, $\mathrm{Km}^{\mathrm{r}}$ & {$[42]$} \\
\hline pDP10 & Derived from pDXW-10, harboring phbCAB operon & This study \\
\hline pDPP10 & Derived from pDP10, harboring $p h b C A B$ operon and $p p n K$ gene & This study \\
\hline pDP01 & Derived from pDXW-10, harboring ppnK gene & This study \\
\hline
\end{tabular}

fermentor (BIOTECH-5BG, Baoxing Co., China) with a working volume of $2.5-1$. The culture condition was set at $30{ }^{\circ} \mathrm{C}$ and $\mathrm{pH} 7.0$ under the $600 \mathrm{rpm}$ agitation speed. The $\mathrm{pH}$ was controlled automatically by addition of $50 \%$ ammonia water. Temperature was also adjusted automatically by the fermentor.

\section{RNA preparation and transcriptional analysis}

Samples for RNA preparation were cultivated for $24 \mathrm{~h}$ in LBG in shake flask at $30{ }^{\circ} \mathrm{C}$. Total cellular RNA was extracted using the RNA simple Total RNA Kit (TIAN GEN, China). Reverse transcription was carried out with the PrimeScript ${ }^{\mathrm{TM}}$ RT reagent Kit (TaKaRa, China) according to the instructions of the manufacturer. The mRNA levels were determined by semi-quantitative reverse transcription (q) RT-PCR using SYBR green PCR master mix (ABI 7000; Applied Biosystems, CA). The 16S rRNA gene was used as an endogenous control. For qRTPCR, 1/20 of each RT-PCR product was used as the template for DNA amplification, using specific primer pairs for each gene. The results of the reactions were processed using specific software (ABI Prism 7000 SDS software). The RT-PCR measurement was repeated three times for each sample. The $\arg B, \arg C, \operatorname{argD}, \operatorname{argF}, \arg G, \operatorname{argH}, \operatorname{argJ}$, $p h b C, p h b A, p h b B$ and $p p n K$ gene transcript primers were listed in Table 6.

\section{Sample preparation for TEM imaging}

For all different samples for TEM imaging were prepared as reported [45]. Bacteria were fixed with glutaraldehyde $2 \%$ and paraformaldehyde $2 \%$ in a buffer of sodium cacodylate $0.1 \mathrm{~mol} / \mathrm{L}(\mathrm{pH} 7.4)$ and then post-fixed in $1 \%$ buffered osmium tetroxide. The bacteria was then completely dehydrated with ethanol at room temperature and then embedded in epoxy resin (polymerization at $60{ }^{\circ} \mathrm{C}$ for $48 \mathrm{~h}$ ). Ultrathin sections of $50 \mathrm{~nm}$ thicknesses were cut with a diamond knife, deposited on copper grids
Table 6 Primers of RT-PCR used in this study

\begin{tabular}{|c|c|}
\hline Primers & Nucleotide sequence $\left(5^{\prime}-3^{\prime}\right)$ \\
\hline \multicolumn{2}{|l|}{$\arg B$} \\
\hline$-F$ & TCGGTGTTGCTGGAGCTTT \\
\hline$-\mathrm{R}$ & TTCCCCATCCTTGTCGTCTT \\
\hline \multicolumn{2}{|l|}{$\arg C$} \\
\hline$-F$ & AGTCCTTGTTACCTCCGCAATC \\
\hline$-\mathrm{R}$ & CTGCTGCCTCATCAAAACCA \\
\hline \multicolumn{2}{|l|}{$\arg D$} \\
\hline$-F$ & CTTGATGTTGGGCGTGGT \\
\hline$-\mathrm{R}$ & GCGTCTGCGATTTCTTCGT \\
\hline \multicolumn{2}{|l|}{$\operatorname{argF}$} \\
\hline$-F$ & ACCACACCTTTCGTTCCTTACC \\
\hline$-R$ & AGGCGGTTTTCTGCTTCATC \\
\hline \multicolumn{2}{|l|}{$\arg G$} \\
\hline$-F$ & TCTCGTGGGCATCAAGTCC \\
\hline$-R$ & TGACATCTTCCAAAGCCTCGT \\
\hline \multicolumn{2}{|l|}{$\operatorname{argH}$} \\
\hline$-F$ & AATCATGCCGCAGAAGAAGAA \\
\hline$-\mathrm{R}$ & GTCAAGGTGGAAACCAAACCA \\
\hline \multicolumn{2}{|l|}{$\arg \rfloor$} \\
\hline$-F$ & GTGAAGGTGAGCCGAGAGAAC \\
\hline$-\mathrm{R}$ & ACCATTACACGCATTAGCATTACC \\
\hline \multicolumn{2}{|l|}{ phbc } \\
\hline$-\mathrm{F}$ & GCGTTCTACCTGCTCAATG \\
\hline$-R$ & GATTGGTGGCAAGGAAGTT \\
\hline \multicolumn{2}{|l|}{ phbA } \\
\hline$-F$ & CAAGGAATACGGCATCACA \\
\hline$-\mathrm{R}$ & CGAACTCGTCGGTCTTGAA \\
\hline \multicolumn{2}{|l|}{ phbB } \\
\hline$-F$ & GACGAGATGTTGACGATGC \\
\hline$-\mathrm{R}$ & GACGAGATGTTGACGATGC \\
\hline \multicolumn{2}{|l|}{ ppnK } \\
\hline$-\mathrm{F}$ & GTCTGACTCACTTGAAGAGGC \\
\hline$-\mathrm{R}$ & GCAACCAAAGGAAGCAAC \\
\hline
\end{tabular}


(mesh 200) and then stained with uranyl acetate aqueous solution $(20 \mathrm{~min})$ and lead citrate $(5 \mathrm{~min})$. Samples were observed at $80 \mathrm{kV}$ with HITACHI H-7650 equipped with a CCD camera.

\section{Analytical methods}

Bacterial growth was monitored by measuring the optical density (OD) at $562 \mathrm{~nm}$ using the spectrophotometer, and the dry cell weight (DCW) was determined by a pre-calibrated relationship $\left(1 \mathrm{OD}=0.375 \mathrm{~g} \mathrm{~L}^{-1} \mathrm{DCW}\right)$. Glucose concentration in the media assayed using a glucose analyzer (Biosensor SBA-50, Shandong, China). The enzyme activities of $\mathrm{PhbC}, \mathrm{PhbA}, \mathrm{PhbB}$ and PpnK were detected by the methods reported after the cells were washed twice with $0.1 \mathrm{M}$ Tris- $\mathrm{HCl}(\mathrm{pH} 7.5)$ and then disrupted by sonication [21, 37]. The concentration of total cellular proteins was determined by the Modified Bradford Protein Assay Kit (Sangon Biotech, Shanghai, China). Bacterial were harvested by centrifugation at $1000 \mathrm{rpm}$ for $10 \mathrm{~min}$, washed twice by distilled water, and then evaporated overnight for the next $\mathrm{PHB}$ content detection. $\mathrm{PHB}$ content was then tested by gas chromatograph (GC) after methanolysis of lyophilized cells in chloroform [46]. Extracellular L-arginine and other related amino acids were measured by an Agilent 1100 HPLC. The metabolic intermediates or by-products in batch cultivation were analyzed by HPLC (SHIMADZU LC-20A) equipped with an ion exchange column (Aminexs HPX-87H, $7.8 \times 300 \mathrm{~mm}$, BioRad). The mobile phase was $5 \mathrm{mM}$ $\mathrm{H}_{2} \mathrm{SO}_{4}$ pumped at a flow rate of $0.5 \mathrm{ml} \mathrm{min}^{-1}$. NADPH and $\mathrm{NADP}^{+}$concentration were detected using $\mathrm{NADP}^{+} /$ NADPH Quantification Colorimetric Kit (BioVision Incorporated, USA). All of the measurements, particularly the most important state variables, were measured in three parallels.

\section{Additional file}

Additional file 1. Assay of enzyme activities of crude PpnK. Samples were taken at $24 \mathrm{~h}$ of the shake flask using LBG culture. NAD kinase encoding by ppnK in C. crenatum contained ATP-NAD ${ }^{+}$kinase, ATP-NADH kinase, PolyP-NAD ${ }^{+}$kinase and PolyP-NADH kinase. Each data represented the average value of three independent measurements.

\section{Abbreviations}

PHB: Poly- $\beta$-hydroxybutyrate; PhbC: PHB synthase; PhbA: $\beta$-Ketothiolase; PhbB: NADPH-dependent acetoacetyl-CoA reductase; ArgC: $\mathrm{N}$-acetylglutamate 5-semialdehyde dehydrogenase; PpnK: NAD kinase; LBG: LB with $0.5 \%$ glucose; TEM: Transmission electron microscope; DCW: Dry cell weight.

\section{Authors' contributions}

$M X$ and ZR participated in the design of the study and performed the statistical analysis. MX and JQ carried out the construction of the strains, enzyme activities determination, participated in the fermentation research and drafted the manuscript. HY participated in detecting the by-products. YG and ZX gave several useful suggestions. XW provided pDXW-10 and pBHR68 for the study. All authors read and approved the final manuscript.

\section{Acknowledgements}

This work was supported by the National Basic Research Program of China (973 Program) (2012CB725202), the High-tech Research and Development Programs of China (2012AA022102, 2015AA021004), the National Natural Science Foundation of China (31300028), the Research Project of Chinese Ministry of Education (113033A), the Jiangsu Provincial National Basic Research Program (BK20130137), the Fundamental Research Funds for the Central Universities (JUSRP51306A), the Project Funded by the Priority Academic Program Development of Jiangsu Higher Education Institutions, the 111 Project (No. 111-2-06), and the Jiangsu province "Collaborative Innovation Centre for Advanced Industrial Fermentation" industry development program.

\section{Competing interests}

The authors declare that they have no competing interests.

Received: 25 October 2015 Accepted: 8 January 2016

Published online: 19 January 2016

\section{References}

1. Granik VG. Metabolism of L-Arginine. Pharm Chem J. 2003;37:111-27.

2. Elias DB, Barbosa MC, Rocha LB, Dutra LL, Silva HF, Martins AM, Gonçalves RF. L-arginine as an adjuvant drug in the treatment of sickle cell anaemia. Br J Haematol. 2013;160:410-2.

3. Hristina K, Langerholc T, Trapecar M. Novel metabolic roles of L-arginine in body energy metabolism and possible clinical applications. J Nutr Health Aging. 2014;18:213-8.

4. Glansdorff N, Xu Y. Microbial arginine biosynthesis: pathway, regulation and industrial production. In: Wendisch $V$, editor. Amino acid biosynthesis pathways, regulation and metabolic engineering, vol. 5. Berlin, Heidelberg: Springer; 2007. p. 219-57 (Microbiology Monographs)

5. Xu M, Rao Z, Yang J, Dou W, Xu Z. The effect of a LYSE exporter overexpression on L-arginine production in Corynebacterium crenatum. Curr Microbiol. 2013;67:271-8.

6. Schneider J, Niermann K, Wendisch VF. Production of the amino acids I-glutamate, L-lysine, L-ornithine and L-arginine from arabinose by recombinant Corynebacterium glutamicum. J Biotechnol. 2011;154:191-8.

7. Ikeda M, Mitsuhashi S, Tanaka K, Hayashi M. Reengineering of a Corynebacterium glutamicum L-arginine and L-citrulline producer. Appl Environ Microbiol. 2009;75:1635-41.

8. Caldara M, Dupont G, Leroy F, Goldbeter A, De Vuyst L, Cunin R. Arginine biosynthesis in Escherichia coli: experimental perturbation and mathematical modeling. J Biol Chem. 2008;283:6347-58.

9. Xu H, Dou W, Xu H, Zhang X, Rao Z, Shi Z, Xu Z. A two-stage oxygen supply strategy for enhanced L-arginine production by Corynebacterium crenatum based on metabolic fluxes analysis. Biochem Eng J. 2009:43:41-51.

10. Xu M, Rao Z, Xu H, Lan C, Dou W, Zhang X, Jin J, Xu Z. Enhanced production of $\mathrm{L}$-arginine by expression of Vitreoscilla hemoglobin using a novel expression system in Corynebacterium crenatum. Appl Biochem Biotechnol. 2011;163:707-19.

11. Xu M, Rao Z, Yang J, Xia H, Dou W, Jin J, Xu Z. Heterologous and homologous expression of the arginine biosynthetic $\arg \mathrm{C} \sim \mathrm{H}$ cluster from Corynebacterium crenatum for improvement of ( $\mathrm{L}$-arginine production. J Ind Microbiol Biotechnol. 2012;39:495-502.

12. Xu M, Rao Z, Dou W, Yang J, Jin J, Xu Z. Site-directed mutagenesis and feedback-resistant N-acetyl-L-glutamate kinase (NAGK) increase Corynebacterium crenatum L-arginine production. Amino Acids. 2012;43:255-66

13. Braunegg G, Lefebvre G, Genser KF. Polyhydroxyalkanoates, biopolyesters from renewable resources: physiological and engineering aspects. J Biotechnol. 1998;65(2-3):127-61.

14. Lee YS. Plastic bacteria Progress and prospects for polyhydroxyalkanoate production in bacteria. Trends Biotechnol. 1996;14:431-8.

15. Harding KG, Dennis JS, von Blottnitz H, Harrison ST. Environmental analysis of plastic production processes: comparing petroleumbased polypropylene and polyethylene with biologically-based poly-beta-hydroxybutyric acid using life cycle analysis. J Biotechnol. 2007; 130:57-66. 
16. Wang Q, Yu H, Xia Y, Kang Z, Qi Q. Complete PHB mobilization in Escherichia coli enhances the stress tolerance: a potential biotechnological application. Microb Cell Fact. 2009;8:9.

17. Gu P, Kang J, Yang F, Wang Q, Liang Q, Qingsheng Q. The improved L-tryptophan production in recombinant Escherichia coli by expressing the polyhydroxybutyrate synthesis pathway. Appl Microbiol Biotechnol. 2013;97:4121-7.

18. Liu Q, Ouyang SP, Kim J, Chen GQ. The impact of PHB accumulation on L-glutamate production by recombinant Corynebacterium glutamicum. J Biotechnol. 2007;132:273-9.

19. Kang Z, Gao C, Wang Q, Liu H, Qi Q. A novel strategy for succinate and polyhydroxybutyrate co-production in Escherichia coli. Bioresour Technol. 2010;101:7675-8.

20. Senior PJ, Dawes EA. The regulation of poly-P-hydroxybutyrate metabolism in Azotobacter beijerinckii. Biochem J. 1973;134:225-38.

21. Li ZJ, Cai L, Wu Q, Chen GQ. Overexpression of NAD kinase in recombinant Escherichia coli harboring the phbCAB operon improves poly(3hydroxybutyrate) production. Appl Microbiol Biotechnol. 2009;83:939-47.

22. Glansdorff N, Xu Y. Microbial Arginine Biosynthesis:pathway, Regulation and Industrial Production. Microbiol Monogr. 2006;5:219-57.

23. Wang Y, San KY, Bennett GN. Improvement of NADPH bioavailability in Escherichia coli by replacing NAD(+)-dependent glyceraldehyde3-phosphate dehydrogenase GapA with NADP(+)-dependent GapB from Bacillus subtilis and addition of NAD kinase. J Ind Microbiol Biotechnol. 2013;40:1449-60.

24. Hk C, Gm W. Regeneration of nicotinamide cofactors for use in organic synthesis. Appl Biochem Biotechnol. 1987;14:147-97.

25. Liu W, Wang P. Cofactor regeneration for sustainable enzymatic biosynthesis. Biotechnol Adv. 2007;25:369-84.

26. Garavaglia S, Raffaelli N, Finaurini L, Magni G, Rizzi M. A novel fold revealed by mycobacterium tuberculosis NAD kinase a key allosteric enzyme in NADP biosynthesis. JBC Papers in Press. 1993;279(39):40980-6.

27. Kawai S, Mori S, Mukai T. Molecular characterization of Escherichia coli NAD kinase. Eur J Biochem. 2001;268(15):4359-65.

28. Kawai S, Suzuki S, Mori S, Murata K. Molecular cloning and identification of UTR1 of a yeast Saccharomyces cerevisiae as a gene encoding an NAD kinase. FEMS Microbiol Lett. 2001;200:181-4.

29. Lerner F, Niere M, Ludwig A, Ziegler M. Structural and functional characterization of human NAD kinase. Biochem Biophys Res Commun. 2001;288:69-74.

30. Grose JH, Joss L, Velick SF, Roth JR. Evidence that feedback inhibition of NAD kinase controls responses to oxidative stress. Proc Natl Acad Sci USA. 2006;103:7601-6.

31. Yin L, Zhao J, Chen C, Hu X, Wang X. Enhancing the carbon flux and NADPH supply to increase L-isoleucine production in Corynebacterium glutamicum. Biotechnol Bioprocess Eng. 2014;19:132-42.

32. Shi A, Zhu X, Lu J, Zhang X, Ma Y. Activating transhydrogenase and NAD kinase in combination for improving isobutanol production. Metab Eng 2013;16:1-10.

33. Lee HC, Kim JS, Jang W, Kim SY. Thymidine production by overexpressing $\mathrm{NAD}^{+}$kinase in an Escherichia coli recombinant strain. Biotechnol Lett. 2009;31:1929-36.
34. Lindner SN, Niederholtmeyer H, Schmitz K, Schoberth SM, Wendisch VF. Polyphosphate/ATP-dependent NAD kinase of Corynebacterium glutamicum: biochemical properties and impact of ppnK overexpression on lysine production. Appl Microbiol Biotechnol. 2010;87:583-93.

35. Shi F, Li K, Huan X, Wang X. Expression of NAD $(H)$ kinase and glucose6-phosphate dehydrogenase improve NADPH supply and L-isoleucine biosynthesis in Corynebacterium glutamicum ssp. lactofermentum. Appl Biochem Biotechnol. 2013;171:504-21.

36. Bhubalan K, Chuah JA, Shozui F, Brigham CJ, Taguchi S, Sinskey AJ, Rha C, Sudesh K. Characterization of the highly active polyhydroxyalkanoate synthase of Chromobacterium sp. strain USM2. Appl Environ Microbiol. 2011;77:2926-33.

37. Steinbuchel PSA, Schlegel DHG. Cloning of the Alcaligenes eutrophus genes for synthesis of poly-beta-hydroxybutyric acid (PHB) and synthesis of PHB in Escherichia coli. J Bacteriol. 1988;170:5837-47.

38. Nishimura T, Saito T, Tomita K. Purification and properties of $\beta$-ketothiolase from Zoogloea ramigera. Arch Microbiol. 1978;116:21-7.

39. Shi F, Huan X, Wang X, Ning J. Overexpression of NAD kinases improves the L-isoleucine biosynthesis in Corynebacterium glutamicum ssp. lactofermentum. Enzyme Microb Technol. 2012;51:73-80.

40. Shigeyuki K, Kousaku M. Structure and function of NAD kinase and NADP phosphatase: key enzymes that regulate the intracellular balance of NAD $(H)$ and NADP(H). Biosci Biotechnol Biochem. 2008;72:919-30.

41. Ando T, Ohashi K, Ochiai A, Mikami B, Kawai S, Murata K. Structural determinants of discrimination of $\mathrm{NAD}^{+}$from $\mathrm{NADH}$ in yeast mitochondrial NADH kinase Pos5. J Biol Chem. 2011;286:9.

42. Xu D, Tan Y, Shi F, Wang X. An improved shuttle vector constructed for metabolic engineering research in Corynebacterium glutamicum. Plasmid. 2010;64:85-91.

43. Magni G, Orsomando G, Raffaell N. Structural and functional properties of NAD kinase, a key enzyme in NADP biosynthesis. Med Chem. 2006;6:739-46.

44. Spiekermann P, Rehm BH, Kalscheuer R, Baumeister D, Steinbuchel A. A sensitive, viable-colony staining method using Nile red for direct screening of bacteria that accumulate polyhydroxyalkanoic acids and other lipid storage compounds. Arch Microbiol. 1999;171:73-80.

45. Mayet C, Deniset-Besseau A, Prazeres R, Ortega JM, Dazzi A. Analysis of bacterial polyhydroxybutyrate production by multimodal nanoimaging. Biotechnol Adv. 2013;31:369-74.

46. Braunegg $G$, Sonnleimer B, Lafferty R. A rapid gas chromatographic method for the determination of poly- $\beta$-hydroxybutyric acidin microbial biomass. European J Appl Microbiol Biotechnol. 1978;6:29-37.

\section{Submit your next manuscript to BioMed Central and we will help you at every step:}

- We accept pre-submission inquiries

- Our selector tool helps you to find the most relevant journal

- We provide round the clock customer support

- Convenient online submission

- Thorough peer review

- Inclusion in PubMed and all major indexing services

- Maximum visibility for your research

Submit your manuscript at www.biomedcentral.com/submit
C Biomed Central 